\title{
Selection, Inheritance, and the Evolution of Parent-Offspring Interactions
}

Judith E. Lock, Per T. Smiseth, and Allen J. Moore

School of Biological Sciences, University of Manchester, Manchester M13 9PT, United Kingdom

Submitted September 11, 2003; Accepted March 26, 2004; Electronically published May 13, 2004

ABSTRACT: Very few studies have examined parent-offspring interactions from a quantitative genetic perspective. We used a crossfostering design and measured genetic correlations and components of social selection arising from two parental and two offspring behaviors in the burying beetle Nicrophorus vespilloides. Genetic correlations were assessed by examining behavior of relatives independent of common social influences. We found positive genetic correlations between all pairs of behaviors, including between parent and offspring behaviors. Patterns of selection were assessed by standardized performance and selection gradients. Parental provisioning had positive effects on offspring performance and fitness, while remaining near the larvae without feeding them had negative effects. Begging had positive effects on offspring performance and fitness, while increased competition among siblings had negative effects. Coadaptations between parenting and offspring behavior appear to be maintained by genetic correlations and functional trade-offs; parents that feed their offspring more also spend more time in the area where they can forage for themselves. Families with high levels of begging have high levels of sibling competition. Integrating information from genetics and selection thus provides a general explanation for why variation persists in seemingly beneficial traits expressed in parent-offspring interactions and illustrates why it is important to measure functionally related suites of behaviors.

Keywords: begging, genetic correlations, parental care, performance gradients, selection gradients, social selection.

Social interactions are a ubiquitous feature of the life history of many organisms, and these interactions can have a dramatic influence on an individual's fitness. When behavior expressed in social interactions influences fitness, a type of selection results that has been termed "social

\footnotetext{
* Corresponding author; e-mail: allen.j.moore@man.ac.uk.
}

Am. Nat. 2004. Vol. 164, pp. 13-24. (C) 2004 by The University of Chicago. 0003-0147/2004/16401-40110\$15.00. All rights reserved. selection" to distinguish it from selection that results from abiotic factors or ecological selection (Crook 1972; WestEberhard 1983; Wolf et al. 1999). Similarly, traits expressed during social interactions, which typically are behaviors, have been termed "interacting phenotypes" (Moore et al. 1997, 1998) because such phenotypes are predicted to follow a different evolutionary trajectory than other morphological or life-history traits (Moore et al. 1997, 1998, 2002; Wolf et al. 1999). This altered evolutionary trajectory occurs because interacting phenotypes can be both an agent and a target of selection. Unlike abiotic factors influencing fitness, this latter characteristic means that because interacting phenotypes can have a heritable basis, they can themselves evolve, which leads to complex evolutionary dynamics (Moore et al. 1997, 1998, 2002). Thus information on both selection and inheritance is particularly important for traits that influence social interactions.

For many organisms, one of the most common and also commonly studied social interactions is the one between parents and offspring. However, most studies of parentoffspring interactions have focused on the benefits and costs of parenting or offspring signaling (Clutton-Brock 1991; Rosenblatt and Snowden 1996; Wright and Leonard 2002). Offspring growth and survival can clearly depend on the amount of resources a parent provides, which in turn may depend on the intensity of offspring signaling. Many fewer studies have quantified patterns of genetic variation and covariation in parent and offspring behaviors expressed during interactions (Kölliker et al. 2000; Agrawal et al. 2001; Kölliker and Richner 2001; Hager and Johnstone 2003). Even more surprising was that, to our knowledge, no studies have provided measures of selection gradients associated with parental care and begging. Yet theoretical work suggests that it is critical to understand the inheritance of parent and offspring behaviors (Cheverud and Moore 1994; Moore et al. 1998) and the nature of selection arising from the effects of care or begging on offspring traits (Wolf and Brodie 1998; Wolf et al. 1999) to fully appreciate how parental care and offspring begging might evolve. 
In this study we set out to empirically determine the nature of inheritance and selection associated with parentoffspring interactions involving care. Our goal was twofold: first, to determine whether there was coadaptation in parent-offspring communication in a species where both parental provisioning of food and begging for food can be quantified directly, and second, to quantify the strength and nature of selection arising from parenting or begging. We used a cross-fostering design to eliminate common environment effects on parenting or begging between relatives, and we examined both genetic correlations and the influence of variation in parent and offspring traits on offspring performance and fitness using the burying beetle Nicrophorus vespilloides as our model. Burying beetles provide a convenient organism to study parental care and offspring begging under nearly natural environments in the laboratory (Eggert and Müller 1997; Scott 1998) and have proven useful in quantitative genetic studies of maternal effects (Rauter and Moore 2002a, 2002b). Crossfostering is easily accomplished in burying beetles (Rauter and Moore 2002a). There are also theoretical reasons to expect variation in responses to care (Rauter and Moore $2002 b$ ) because burying beetles are not completely dependent on care. Offspring can either beg or self-feed, and $N$. vespilloides has been described as a "partially begging" species (Smiseth and Moore 2002, 2004a, 2004b; Smiseth et al. 2003).

We first tested the hypothesis that parental care and offspring begging coevolve; that is, there is a genetic correlation between the two reflecting coadapted levels of expression (Wade 1998; Wolf and Brodie 1998; Wolf 2000). Next, we tested the hypothesis that parental and offspring behavior result in selection by quantifying how parental behavior associated with care and offspring behavior associated with begging influence offspring growth and development (i.e., we calculated performance gradients; Arnold 1983). We also quantified selection by examining the association between growth and development with fitness (i.e., we calculated selection gradients; Lande and Arnold 1983). Calculation of performance and associated selection (fitness) gradients allows us to measure adaptive significance directly (Arnold 1983). We therefore adopted this quantitative genetic approach to both selection and inheritance to provide an analysis of parent-offspring interactions based on formal evolutionary theory involving multivariate selection (Arnold 1983; Lande and Arnold 1983; Brodie et al. 1995). To the best of our knowledge, such an integrated approach quantifying both patterns of inheritance and the strength and pattern of multivariate selection has not been attempted in a single study.

\section{Material and Methods}

Burying Beetle Basic Biology

Burying beetles have a rapid generation time and welldescribed behavior and ecology (comprehensively reviewed in Eggert and Müller 1997; Scott 1998). Furthermore, burying beetles are easily reared in the laboratory under conditions very similar to nature, making them excellent subjects for investigations of parental care, genetics, and evolution. Burying beetles breed on vertebrate carcasses, which form the sole food resource for offspring. Carcasses can be located either by males or by females. If a male locates the carcass, he emits pheromone to attract a female. If a female locates a carcass, she lays eggs some distance from the carcass. The female, or male and female together, then remove fur or feathers, roll the carcass into a ball, keep it free of fungus and bacteria, and prepare a cavity in the top of the carcass from which the offspring forage (self-feed) from partially digested meat. The parents can also forage for themselves from the carcass from this cavity area while they are partially digesting the carcass for the offspring (Scott and Gladstein 1993; S. Musa, P. T. Smiseth, and A. J. Moore, unpublished data).

At $20^{\circ} \mathrm{C}$, larvae hatch approximately $60 \mathrm{~h}$ after the eggs are laid (P. T. Smiseth and A. J. Moore, unpublished data), and they crawl to the prepared carcass. Once they reach the carcass, Nicrophorus vespilloides larvae require direct provisioning of food from their parents for the first $12 \mathrm{~h}$, with a decreasing reliance after that (Eggert et al. 1998). Parental care in burying beetles goes well beyond providing previously secured food for the larvae and involves direct and detailed social interactions between parents and offspring. Larvae can either forage for themselves (self-feed) or beg for food from the parents (Smiseth and Moore 2002, 2004b; Smiseth et al. 2003). Begging can stimulate direct parental provisioning, which occurs by regurgitation of predigested carrion into the mouth of the larvae and is indicated by mouth-to-mouth contact between the parent and a larva (Rauter and Moore 1999, in press; Smiseth et al. 2003; Smiseth and Moore 2004a, 2004b; P. T. Smiseth, C. T. Darvell, and A. J. Moore, unpublished manuscript). Parents never feed larvae unless they are begging. Parental care in Nicrophorus is consistent with most common expectations for parental care reflecting our mammalian bias and with studies of avian parental care (Rauter and Moore 1999).

Development of $N$. vespilloides larvae is rapid, and individuals reach the adult stage approximately $30 \mathrm{~d}$ after they hatch. In $N$. vespilloides, larvae feed from the carcass for $5-7 \mathrm{~d}$ although parental care is variable and decreasing over this time. Begging ceases after $72 \mathrm{~h}$, although parents continue to visit the crater until the larvae disperse (Smiseth et al. 2003). Once the carcass has been consumed, 
larvae disperse by crawling from the remains of the carcass, and they have no more contact with the parent. At this point, larvae have completed feeding and growth and enter a "wandering stage" for 7-10 d. These wandering larvae then bury themselves in the soil and pupate, emerging to adulthood after an additional 7-10 d. Adults are sexually mature 7-10 $\mathrm{d}$ after they emerge.

Although we conducted our study in the laboratory, we maintained conditions to resemble those in the field as closely as possible. Behavior expressed in the laboratory and field behavior are very similar (Scott 1998; Beeler et al. 1999). These beetles reproduce underground where temperature fluctuations should be minimized and the carcass can be relatively well hidden from predators and competitors. The lack of predators and competitors in our laboratory conditions may affect larval survival after dispersal from the carcass, when the larvae burrow underground and pupate under the soil. However, mortality due to predation after dispersal would not reflect direct fitness effects of the interaction with parents, which was the focus of our study.

\section{Experimental Design}

Smiseth and Moore (2002) provide details on the field origin (Wales) and laboratory husbandry of the beetles studied. More than 100 beetles were collected from the field and maintained as outbred stock for several generations in the laboratory. For the current study, 34 independent breeding pairs were established from newly emerged, randomly selected, nonsibling male and female virgin beetles. When the beetles reached sexual maturity, they were set up in a clear plastic container containing 1$\mathrm{cm}$-depth damp soil and placed in an observation room lit with red light. The following day the pair was provided with a 10-17-g mouse carcass, fresh frozen and thawed before the experiment (supplied by Livestock Direct, Sheffield). The mouse was provided 3-4 h before "sunset" (=lights off), a time when $N$. vespilloides typically search for carrion in nature (Kocárek 2001). The carcass was checked for the presence of larvae twice a day.

There were two rearing treatments for each family: offspring reared by a related female (the biological mother as the caretaker) or by an unrelated female (a foster mother as the caretaker). Burying beetles use temporal cues in kin recognition (time of arrival of larvae to the carcass; Müller and Eggert 1990), which facilitates cross-fostering. In these experiments each female produced and reared two broods (one as a biological mother and one as a foster mother). Larvae that arrived at the carcass within 24 h (i.e., first instars) were removed, counted, and returned to the carcass maintained by their natal mother or that of an unrelated foster mother whose larvae hatched at the same time. The order of the related or unrelated caretaker treatments was randomized for each family.

Once the family units were established, the carcasses and females were moved to new boxes with fresh soil in order to prevent the arrival of any additional larvae after the manipulation. At this time, the male was also removed so that behavioral observations were of female uniparental care only. The male was removed because uniparental and biparental care have equivalent effects on offspring fitness (Smiseth et al., in review). The manipulated broods were left for a further $24 \mathrm{~h}$, and observations of larval begging and parental provisioning were then carried out when the larvae were $\sim 48 \mathrm{~h}$ old and second instars.

\section{Measures of Behavior}

In this study we were interested in the covariances between natural levels of variation in direct parental care for the offspring and offspring performance (or fitness) rather than manipulating parental care as has been done previously (e.g., Eggert et al. 1998; Rauter and Moore 2002b). Variations in parental behavior that did not involve direct interactions with the larvae (parental defense of the carcass, maintenance of the carcass) may be important influences on offspring fitness, but they are beyond the scope of this study, which focused specifically on interacting phenotypes of parental and offspring behavior. Therefore, for this experiment we focused on behaviors expressed when parents and larvae are in close proximity (defined as within 1 pronotum length or less away from the larvae) and thus must involve an interaction. Close proximity is required for offspring begging; that is, offspring never beg to attract a parent because begging occurs only if parents are next to the offspring (Rauter and Moore 1999; Smiseth and Moore 2002).

Our measures of both parental and offspring behaviors are identical to those we have used in previous studies (Smiseth and Moore 2002, 2004a, 2004b; Smiseth et al. 2003). Two parental behaviors were examined; the first was the percentage of time the parent spent in the cavity in close proximity to the offspring but without feeding the offspring. This behavior was recorded because parents can forage for themselves when they are in the cavity (Scott and Gladstein 1993). The second behavior was the percentage of time the parent spent directly provisioning the offspring with food when they were in close proximity. Begging is a necessary but sufficient trait to elicit provisioning. Two larval traits that indicate levels of larval begging were scored during our behavioral observations, the first being the average percent of time spent begging by each larva in the brood when the parent was present and in close proximity. Larvae start begging only when the adult is at a distance that corresponds approximately to 
the width of the parent's pronotum (Rauter and Moore 1999; Smiseth and Moore 2002). The percent of time spent begging is therefore a measure of the average begging effort by each larva in the brood (Smiseth and Moore 2004a). The second trait indicator was the mean number of larvae begging in scans where at least one larva was begging. This measure reflects the proportion of siblings that, at a given time, are competing for food from the parent (Smiseth and Moore 2002).

Parental and larval behaviors were recorded using instantaneous scan sampling (Martin and Bateson 1986). Observations lasted for $30 \mathrm{~m}$ and were made $24 \mathrm{~h}$ after larvae arrived on the carcass. We chose this period for observation because, although there is consistent behavior within families (A. J. Moore, unpublished data), this is the day when there is the highest level of interaction between parents and larvae (Smiseth et al. 2003).

\section{Coadaptation of Parenting and Begging}

We adapted a method similar to that of Kölliker et al. (2000; see also Agrawal et al. 2001) and used a crossfostering design to examine the potential genetic covariance between parent-offspring interactions independent of family environment. The main difference between our study and these previous studies is that we measured the amount of begging or amount of parenting in direct response to an interaction with a caretaker rather than relying on an indirect measure of begging either inferred from offspring performance (Agrawal et al. 2001) or measured from artificial recordings (Kölliker et al. 2000). This latter study also measured parental provisioning as a response to manipulated playbacks.

The cross-fostering method we used eliminates the environmental influences of parents on offspring and vice versa by eliminating sources of variation caused by a shared environment. With this design, begging behavior of offspring is measured when an unrelated caretaker rears them, and parenting of caretakers is measured when they interact with unrelated larvae. Any covariance between relatives must then reflect genetic influences because the only remaining common influence is shared inheritance. This measure may be inflated by additive components of epistasis, but using parent-offspring covariances eliminates dominance components of variation and so should be close to the additive genetic correlation. We therefore estimated this genetic correlation using a Pearson productmoment correlation between behaviors of relatives expressed in independent social contexts. Our complete design, where parents also took care of their biological offspring, allowed us to compare traits expressed in family and cross-fostered groups as well. Within-social environment correlations (i.e., parents taking care of their own offspring) will differ from between-social environment correlations (i.e., parents interacting with unrelated offspring) by an amount that reflects common environmental influences, thereby permitting a further estimate of how common environment inflates family resemblance in interacting phenotypes.

\section{Measures of Offspring Fitness and Performance}

We scored components of offspring fitness as survival from one life-history stage to another until adult emergence. We also measured offspring performance based on traits that we know can be influenced by parental care (Rauter and Moore 2002a, 2002b; Smiseth et al. 2003) and that we predicted would be associated with fitness because they are fundamental life-history characters: mass of a larva at dispersal from the carcass (i.e., the point at which larval foraging and parental care ceased), the duration of time the larvae stayed on the carcass before dispersing as wanderers, the duration of the developmental stage from dispersal to entering the pupal stage (i.e., the wandering stage where larvae disperse off the resource and no longer forage), and the duration of the pupal stage (i.e., from entering the pupal stage until emergence as an adult beetle). For duration of time the larvae spent on the carcass, there was no variation within families because all larvae disperse from the carcass at the same time.

\section{Quantifying Selection Arising from Parental and Offspring Behavior: Selection and Performance Gradients}

We used linear regression models to calculate standardized selection gradients (Lande and Arnold 1983) that affect life-history traits associated with larval performance. We also calculated performance gradients (Arnold 1983) relating parental and offspring behavior to offspring traits. This two-step process is necessary because, although we can measure the life-history characters or fitness of individuals, we can only measure the behavior of parents toward entire families and the average amount of begging for the brood because individually identifiable marks on the larvae, either natural or applied, do not persist between larval stages (molts). However, analyzing parental performance gradients, offspring performance gradients, and fitness (selection) gradients allows us to integrate all of our measures in a formal evolutionary framework and model (fig. 1), put our work in terms that are used in equations for evolutionary change, and compare relative strengths of the effects (Arnold 1983; Lande and Arnold 1983; Brodie et al. 1995).

Survival from one life-history stage to the next was scored as 0 (died) or 1 (survived), and fitness was converted to relative fitness (Lande and Arnold 1983). Relative 


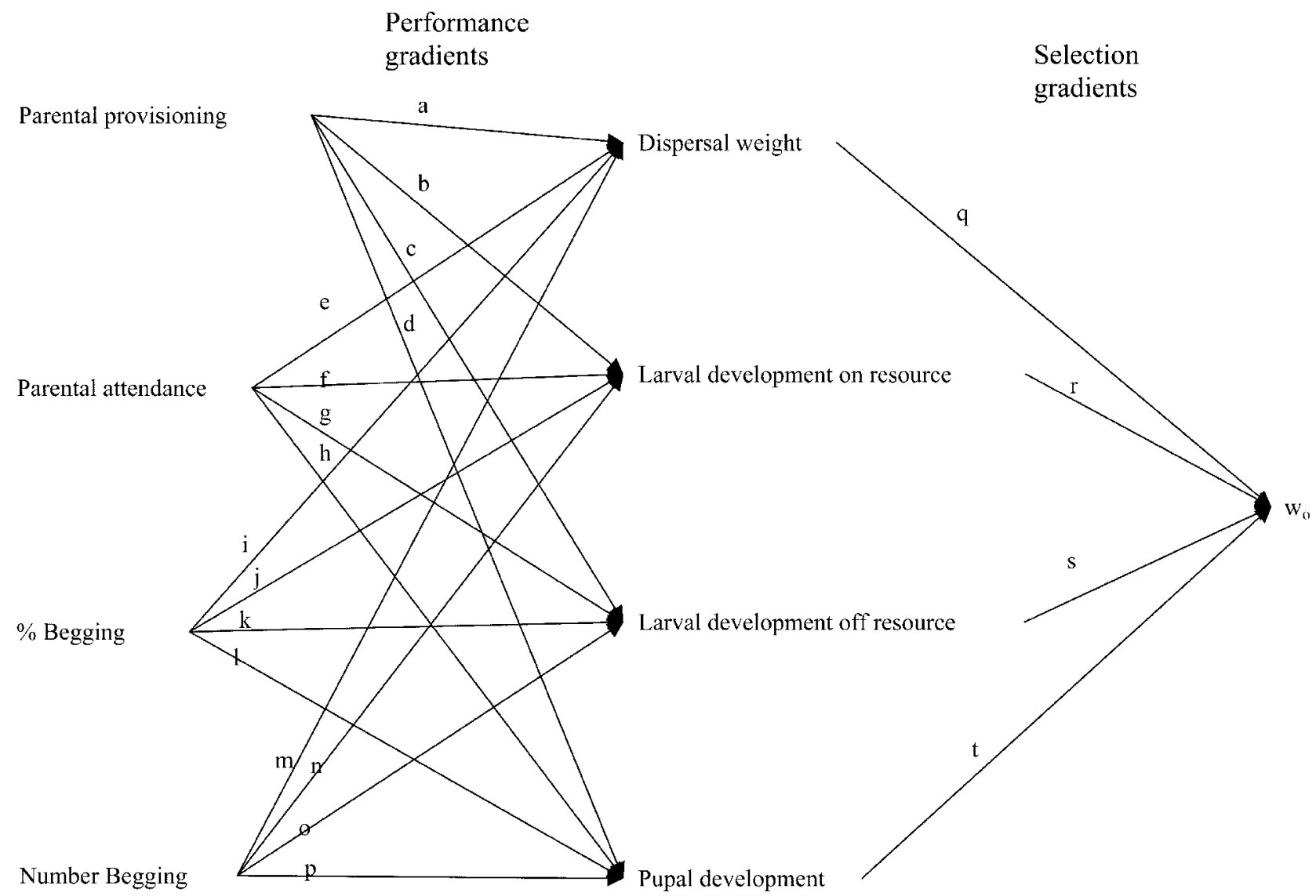

Figure 1: Model of social selection arising from parent-offspring interactions that was tested in Nicrophorus vespilloides. Paths $a-h$ are parental performance gradients $\left(\beta_{\mathrm{f} z}\right.$ : the direct effect of parental care behavior $z_{\mathrm{p}}$ on offspring performance $f$, measured as growth and development), paths $i-p$ are offspring performance gradients $\left(\beta_{\mathrm{fz}}\right.$ : the effects of offspring begging behavior $z_{\mathrm{o}}$ on offspring performance $\left.f\right)$, and paths $q-t$ are standardized linear selection gradients $\left(\beta_{\mathrm{wf}}\right.$ : the fitness gradient arising from the influence of the offspring performance trait $f$ on relative fitness [ $w_{\mathrm{o}}$ ], measured by survival). Values for paths $q-t$ are presented in table 2; values for paths $a-p$ are presented in table 4 . See Arnold (1983) for interpretations of and methods of analysis for performance gradients and Lande and Arnold (1983) for interpretations of and methods of analysis for standardized selection gradients.

fitness was regressed on values of standardized life-history characters (mean $=0, S D=1$ ) using general linear models in SYSTAT to generate selection gradients (Lande and Arnold 1983; Brodie et al. 1995). Significance was determined using logistic regression. Associations between offspring life-history traits were investigated with Pearson product-moment correlation, and significance was determined using Bonferroni corrections. The association between survival and relatedness of caretaker (biological or foster mother) was tested using a contingency test.

Performance gradients were calculated by regressing parent family averages for offspring life-history characters on all measured parental and offspring behaviors. Effects of parental and offspring behavior on each performance trait in offspring were investigated with a separate regression. Average values of the standardized offspring perfor- mance characters were used to avoid inflating degrees of freedom because there was a single behavioral measure for each family.

\section{Statistical Controls}

Our interest was restricted to the effects of social interactions on offspring traits, and we therefore experimentally minimized the potential influence of abiotic traits known to influence burying beetle behavior (Smiseth and Moore 2002). Given the model of social selection that we were testing (fig. 1), we did not wish to include abiotic factors as covariates in our analyses because factors such as brood size and resource size are not properties of individuals but may still influence resemblances among individuals (Wolf et al. 1999). We therefore sought to experimentally min- 
imize any covariances that might be induced by minimizing the variation in these factors.

Providing a mouse of similar size to all families (mean $=13.2 \mathrm{~g}, \mathrm{SD}=2.0$ ) should minimize variation in the amount of resource available to affect parent and offspring behavior. To confirm that our experiment controlled for this factor, we also examined the influence of carcass size statistically. A linear regression of carcass size on the parental behaviors confirmed that the range of carcass sizes we used had no significant effect on percent of time parents spent near larvae $\left(R^{2}<0.001, F=0.005\right.$, $\mathrm{df}=1,64, P=.942)$ or the percent of time spent provisioning $\left(R^{2}<0.001, F=0.049\right.$, df $\left.=1,64, P=.825\right)$. A linear regression of carcass size on offspring behaviors further confirmed that carcass size had no significant effect on either number of begging larvae $\left(R^{2}=0.004, F=\right.$ 0.282 , df $=1,63, P=.598)$ or the percent of time spent begging by each larva $\left(R^{2}=0.002, F=0.170, \mathrm{df}=\right.$ $1,62, P=.681)$ or on brood size $\left(R^{2}=0.010, F=\right.$ 0.640 , df $=1,64, P=.427)$. These results are consistent with previous studies that show that carcass size has little effect on parent or offspring behavior (Smiseth and Moore 2002, 2004a).

Rates of parental provisioning to offspring increase with very large differences in brood size (range 5-25 larvae; Rauter and Moore, in press). We therefore minimized variation in brood size as much as possible (mean $=10.6$ larvae, SD = 4.7), matching brood size for fostered and natal families. We confirmed the success of this approach with linear regression. We found that the brood sizes we used in this study had no significant effect on either of the parental behaviors (percent of time near larvae: $R^{2}=0.017, F=1.129, \mathrm{df}=1,64, P=.292$; percent of time provisioning larvae: $R^{2}=0.001, F=0.056, \mathrm{df}=$ $1,64, P=.813)$.

Brood size can influence begging in $N$. vespilloides as well, but the influence is complex (Smiseth and Moore 2002). There is a curvilinear relationship between brood size and begging, with increasing levels of begging in very small broods $(<5)$ or decreasing levels of begging in large broods $(>35)$. Our brood sizes did not include these extremes, so any effects were expected to be minor. Supporting this, there was no significant linear relationship between total number of observations where begging was observed $\left(R^{2}=0.002, F=0.105, \mathrm{df}=1,64, P=.747\right)$ or total number of begging acts observed $\left(R^{2}=0.019\right.$, $F=1,257, \mathrm{df}=1,64, P=.266)$. Nonetheless, in order to maintain consistency between studies, we used the same measures of begging as we have in other studies (Smiseth and Moore 2002, 2004a, 2004b; Smiseth et al. 2003) where brood size is in the denominator of the behavioral measures of begging.

Each female cared for two different broods, and al- though the order of caring for related or unrelated broods was randomized, females may behave differently when inexperienced or experienced as a parent. To check for this, we used repeated-measures analysis of variance to compare the parental care provided to the first and second clutches. Clutch order was found to have no significant effect on time spent in proximity of larvae $(F=0.023$, df $=$ $1,32, P=.880)$ or provisioning of the larvae $(F=$ $0.220, \mathrm{df}=1,32, P=.643)$.

\section{Results \\ Coadaptation of Parenting and Begging}

Parents and offspring performed the same behaviors regardless of whether they were interacting with relatives or nonrelatives, and the patterns of correlations between behavioral acts were similar (table 1). Begging and provisioning within biological families was more strongly correlated than between unrelated individuals ( $r=0.63$ vs. $r=0.53$ ), although this is not a statistically significant difference $\left(t_{\mathrm{s}}=0.986, P>.5\right)$. In general, common environmental effects did not increase resemblance among relatives much beyond the effects of inheritance (table 1), and in some cases the effects appear to decrease the resemblance among relatives (i.e., the correlation between average number of begging larvae and percent of time the parent spent in the cavity and the number of begging larvae and average percent time spent begging by a larvae). Furthermore, all of the correlations between relatives but controlling for common social experience are significantly different from 0 , suggesting that all social behaviors are influenced by genetics and that there is a genetic covariance between all combinations of parenting and begging traits. For example, investigating the hypothesized genetic relationship between begging and provisioning, there was a strong positive relationship within families between begging to an unrelated caretaker and parental provisioning of food to unrelated larvae (fig. 2). Thus, families with high levels of begging have parents that show high levels of provisioning to the larvae, indicating a strong positive genetic correlation $(r=0.51, \mathrm{SE}=0.09, P=.003)$. This joint expression of begging and provisioning appeared to be beneficial; individuals reared by their biological mothers were more likely to survive than larvae reared by a foster mother $\left(\chi^{2}=13.308\right.$, df $\left.=1, P<.001\right)$.

\section{Quantifying Selection Arising from Parental and Offspring Behavior: Selection and Performance Gradients}

There is strong selection associated with growth and development of larvae (table 2). There was selection for larger size at dispersal and faster larval development, both on 
Table 1: Family mean correlations \pm SE and levels of significance among parental and offspring behaviors

\begin{tabular}{|c|c|c|c|c|}
\hline Behavior & $\begin{array}{l}\text { Percent time a parent } \\
\text { spent in the cavity }\end{array}$ & $\begin{array}{l}\text { Percent time spent } \\
\text { provisioning larvae }\end{array}$ & $\begin{array}{l}\text { Average number of } \\
\text { larvae begging }\end{array}$ & $\begin{array}{l}\text { Percent time spent } \\
\text { begging by a larva }\end{array}$ \\
\hline $\begin{array}{l}\text { Percent time a parent } \\
\text { spent in the cavity }\end{array}$ & & $\begin{array}{c}.551 \pm .084 \\
(P<.001)\end{array}$ & $\begin{array}{l}.511 \pm .088 \\
(P=.003)\end{array}$ & $\begin{array}{c}.559 \pm .084 \\
(P<.001)\end{array}$ \\
\hline $\begin{array}{l}\text { Percent time spent } \\
\text { provisioning larvae }\end{array}$ & $\begin{array}{c}.730 \pm .065 \\
(P<.001)\end{array}$ & & $\begin{array}{c}.545 \pm .085 \\
(P<.001)\end{array}$ & $\begin{array}{l}.505 \pm .089 \\
(P=.003)\end{array}$ \\
\hline $\begin{array}{c}\text { Average number of } \\
\text { larvae begging }\end{array}$ & $\begin{array}{l}.199 \pm .113 \\
(P=.284)\end{array}$ & $\begin{array}{l}.484 \pm .091 \\
(P=.006)\end{array}$ & & $\begin{array}{l}.461 \pm .092 \\
(P=.008)\end{array}$ \\
\hline $\begin{array}{l}\text { Percent time spent } \\
\text { begging by a larva }\end{array}$ & $\begin{array}{c}.744 \pm .064 \\
(P<.001)\end{array}$ & $\begin{array}{c}.634 \pm .076 \\
(P<.001)\end{array}$ & $\begin{array}{l}.124 \pm .118 \\
(P=.499)\end{array}$ & \\
\hline
\end{tabular}

Note: Below the diagonal are correlations between behaviors expressed within genetic families (i.e., by relatives in a shared social environment). Above the diagonal are correlations between behaviors of relatives expressed in an independent social environment (i.e., in a cross-fostered environment and therefore in an independent social environment). SE calculated for Pearson product-moment correlation as in Sokal and Rohlf (1995). Bold type $=$ significant with Bonferroni correction.

and off the carcass (table 3). In addition to the linear selection on the duration of wandering, or larval development off the carcass, there was significant nonlinear selection associated with selection against extremes (table $3)$. Nonlinear selection components were not significant for either mass at dispersal or duration of larval development on the resource.

There were significant correlations among all four of our offspring performance measures except between dispersal weight and duration of larval development on the resource. Combining the two periods of larval development, on and off the carcass, there was a highly significant negative correlation between total larval development and pupal development $(r=-0.206, N=611$, $P<.001)$. Shorter larval development is thus offset by longer pupal development-a trade-off that was seen in another burying beetle species in which it has a genetic basis (Rauter and Moore 2002a, 2002b). Controlling for body mass at dispersal, the partial correlation between larval development and pupal development was even stronger $(r=-0.267)$.

Maternal and offspring performance gradients suggest a consistent pattern of trade-offs in parental and offspring behavior (table 4). In all cases, there was a positive relationship between levels of provisioning and offspring performance but a negative relationship between time spent in the crater without provisioning and offspring performance (table 4). This same pattern of balance between offspring behaviors was seen as well, with a positive effect of time spent begging but a negative effect of number of offspring begging on offspring performance (table 4). However, parental and offspring behavior appear to influence different offspring performance characters.
Parental performance gradients were largest in relation to offspring development (table 4). Increased provisioning significantly reduced the duration of time larvae spent on the carcass, decreased the time spent as wandering larvae off the resource, and significantly increased time as pupae (table 4). In contrast, increased time spent by the parent

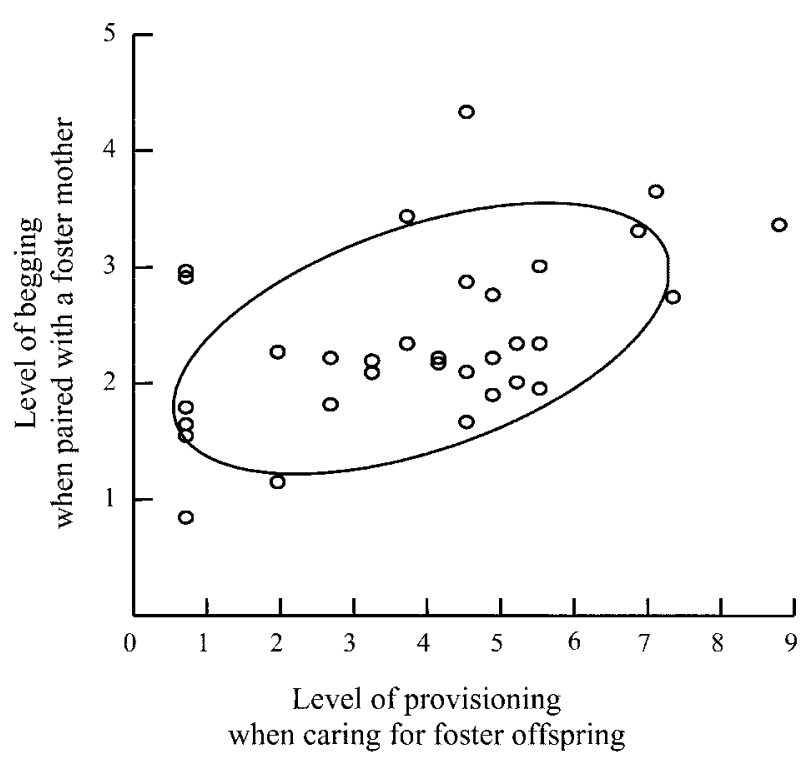

Figure 2: Relationship between provisioning by mothers, measured as percent time spent provisioning unrelated offspring, and begging by offspring, measured as percent time spent begging by larvae to an unrelated parent. The $95 \%$ density ellipse is indicated on the figure, and the positive correlation between these traits $(r=0.51 \pm 0.09)$ is highly significant $(P=.003)$. 
Table 2: Linear $\left(\beta_{\mathrm{w} f i}\right)$ and nonlinear $\left(\gamma_{\mathrm{w} f i}\right)$ standardized selection gradients (Lande and Arnold 1983) relating the effect of offspring performance characters on relative fitness $(w)$ in Nicrophorus vespilloides

\begin{tabular}{|c|c|c|c|c|c|}
\hline $\begin{array}{l}\text { Offspring performance } \\
\text { character }\left(f_{i}\right)\end{array}$ & $N$ & $\begin{array}{l}\text { Linear relationship } \\
\qquad(\beta \pm \mathrm{SE})\end{array}$ & $P$ & $\begin{array}{l}\text { Nonlinear relationship } \\
(\gamma \pm \mathrm{SE})\end{array}$ & $P$ \\
\hline Mass at dispersal & 737 & $.055(.017)$ & .002 & $-.012(.011)$ & .143 \\
\hline $\begin{array}{l}\text { Duration of larval stage } \\
\text { on the resource }\end{array}$ & 737 & $-.049(.017)$ & .005 & $.022(.010)$ & .805 \\
\hline $\begin{array}{l}\text { Duration of larval stage } \\
\text { off the resource }\end{array}$ & 668 & $-.093(.014)$ & $<.001$ & $-.021(.006)$ & $<.001$ \\
\hline
\end{tabular}

in the cavity without provisioning had the opposite effects on development, although only the partial regression between time in the cavity and duration of time spent as wandering larvae was significant (table 4). There was not a significant relationship between parental behaviors and differences in larval mass at dispersal (table 4).

In contrast to parental behavior, offspring performance gradients were largest in relation to larval mass (table 4). Increasing the amount of time a larva spent begging had a positive effect on the final mass of larvae, while increasing numbers of begging larvae had a negative effect on larval mass. The number of begging larvae also had significant negative influences on development. An increase in the number of begging larvae increased the time spent as a wandering larvae off the resource and decreased the time as pupae, both of which are negatively related to fitness (tables 3,4$)$. The amount of time a larva begged was not significantly related to changes in development, although the pattern of association remained consistent (table 4).

\section{Discussion}

This study is unique in simultaneously investigating the role of both selection and inheritance on parent-offspring interactions. We are therefore able to provide a more complete picture of how such interactions can evolve. We show that differences in the expression of parental and offspring behavior in Nicrophorus vespilloides persisted regardless of the social environment in which they were expressed (table 1). There were significant positive correlations between parent and offspring behavior related to the biological origins of the individuals assayed but independent of the social environment within which the behavior was performed, demonstrating that there was significant genetic covariation underlying pairs of parent and offspring behavioral traits. We found significant natural selection on offspring performance (table 2) and corresponding parental and offspring performance gradients arising from parental and offspring behavior, respectively, affecting offspring performance (table 4). Thus, our study places par- ent and offspring interactions within the framework of formal quantitative genetic theory and allows us to interpret patterns of selection and potential inheritance (Arnold 1983). Specifically, the environment-independent correlations support quantitative genetic theories of parentoffspring coadaptation (Wolf and Brodie 1998; Wolf 2000), and the selection and performance gradients suggest hypotheses for how joint expression levels of provisioning and begging may be adaptive in this system.

\section{Coadaptation of Parenting and Begging}

Similar to Agrawal et al.'s (2001) work on burrower bugs, and Hager and Johnstone's (2003) work on inbred strains of laboratory mice, we found that offspring fitness was highest when they were reared by parents that share genes in common with the offspring because of a better match between genetically influenced parent and offspring behavior, supporting a coadaptation between parent and offspring behavior. In addition, there was the expected genetic correlation between parent and offspring traits in N. vespilloides, given a coadaptation (table 1). This correlation was positive-genetic influences led to increased levels of provisioning in parents and increased levels of begging in larvae. The positive genetic correlation is in contrast to Agrawal et al. (2001), who found a negative genetic correlation, but it is consistent with the positive correlation reported by Kölliker et al. (2000) in great tits and suggested by the results of Hager and Johnstone (2003). However, there are positive correlations for all pairs of parent and offspring behaviors, suggesting that it is not possible for individuals to maximize interactions with potentially positive fitness consequences while minimizing interactions that have potentially negative fitness consequences (see below) because negative genetic correlations would be expected if there was an evolved balance of positive and negative effects of interactions. Evidence for why parentoffspring behavior reflects a coadaptation, then, requires the information from our analysis of selection and performance gradients. 
Table 3: Correlations among offspring performance characters

\begin{tabular}{|c|c|c|c|c|}
\hline & $\begin{array}{c}\text { Duration of larval } \\
\text { stage on the resource }\end{array}$ & $\begin{array}{c}\text { Duration of larval } \\
\text { stage off the resource }\end{array}$ & $\begin{array}{l}\text { Duration of } \\
\text { pupal stage }\end{array}$ & Adult size (pronotum) \\
\hline Dispersal weight & $\begin{array}{c}.046, N=737 \\
P=.211\end{array}$ & $\begin{array}{c}.087, N=668 \\
P=.025\end{array}$ & $\begin{array}{c}.315, N=611 \\
P<.001\end{array}$ & $\begin{array}{c}.553, N=601 \\
P<.001\end{array}$ \\
\hline $\begin{array}{l}\text { Duration of larval stage } \\
\text { on the resource }\end{array}$ & & $\begin{array}{c}.119, N=668 \\
P=.002\end{array}$ & $\begin{array}{c}-.175, N=611 \\
P<.001\end{array}$ & $\begin{array}{c}.216, N=601 \\
P<.001\end{array}$ \\
\hline $\begin{array}{l}\text { Duration of larval stage } \\
\text { off the resource }\end{array}$ & & & $\begin{array}{c}-.160, N=611 \\
P<.001\end{array}$ & $\begin{array}{c}.170, N=601 \\
P<.001\end{array}$ \\
\hline $\begin{array}{l}\text { Duration of pupal } \\
\text { stage }\end{array}$ & & & & $\begin{array}{c}.097, N=601 \\
P=.017\end{array}$ \\
\hline
\end{tabular}

Note: Bold type $=$ significant with Bonferroni correction.

Quantifying Selection Arising from Parental and Offspring Behavior: Selection and Performance Gradients

The offspring performance traits we measured were strongly related to fitness in N. vespilloides (table 2). There was selection for larger offspring and those that progressed faster through the larval stage. In addition, there was a negative correlation between the duration of the larval and pupal stages, suggesting that it is advantageous to spend as much time as possible in the pupal rather than the larval stage (table 3 ). These results are consistent with a previous study of Nicrophorus pustulatus (Rauter and Moore 2002b), which found evidence for a similar tradeoff between components of offspring development maintained by negative genetic correlations between developmental stages in N. pustulatus. Overall, heavier larvae (and ultimately larger adults) took longer to develop (table 3), suggesting there may be an upper limit to selection for reduced development time because of trade-offs between components of development. Shifting development to different stages may ameliorate this constraint. Finally, selection on offspring performance traits was not strictly directional (table 2). Although the relationship between larval size and fitness was linear, there was a significant nonlinear relationship between fitness and the duration of development. This further supports the idea of an upper limit to the rate of offspring development.

Given the strength and pattern of selection on offspring performance, the offspring performance gradients for the two begging behaviors we measured appeared to have conflicting function or influence (table 4). The average time spent begging to a parent by each larva in the brood had a positive impact on offspring performance, increasing larval body mass at dispersal and thus adult size at eclosion. This supports a signaling function for begging that is positively related to fitness because only begging offspring are fed by the parents (Smiseth and Moore 2004b). In contrast, there was a negative performance gradient for the mean number of larvae begging during each begging bout for both dispersal weight and the duration of pupal development. These negative fitness effects are likely to arise from intrabrood competition for access to food provisioned by the parent (e.g., Stockley and Parker 2002; Wright and Leonard 2002) because the number of begging offspring is a measure of the number of siblings that, at a given time, are competing for food from the parents (Smiseth and Moore 2002). While this may also reflect an influence of brood size-larger broods may exhibit more competition-the effect is the same. More competition results in a negative effect on offspring performance.

Parental performance gradients show a similar pattern to offspring performance gradients, but the effects are on different offspring characters. In general, provisioning is beneficial because there were significant performance gradients for rate of larval development both on and off the resource. Parental provisioning appears to be a superior way for offspring to obtain food, given that offspring can forage for themselves as well (see also Smiseth and Moore 2004b). Experiments with other burying beetles, where offspring development was compared under conditions where care was provided or eliminated, have also shown that care positively influences larval growth and development (Eggert et al. 1998; Anduaga and Huerta 2001; Rauter and Moore 2002b). Thus, while a positive effect of parental provisioning is unsurprising, this is the first study to quantify the effects of natural levels of variation in care. This positive effect is directly related to feeding and not a function of other social interactions between parents and offspring. Increased time that a parent spent near larvae without feeding them was negatively related to rate of larval development. This is probably because parents may forage for themselves when near the opening to the cavity where food is readily available, as well as regurgitate food 
Table 4: Performance gradients $\left(\beta_{f z i}\right)$ relating parental care behaviors and offspring begging behaviors to offspring performance measured as growth (mass) or rate of development

\begin{tabular}{|c|c|c|c|c|}
\hline \multirow[b]{2}{*}{$\begin{array}{l}\text { Offspring performance } \\
\text { character }(f)\end{array}$} & \multicolumn{4}{|c|}{ Social behavior of parents and offspring $\left(z_{i}\right)$} \\
\hline & $\begin{array}{c}\text { Percent time spent } \\
\text { provisioning larvae } \\
\beta_{f z 1}(\mathrm{SE})\end{array}$ & $\begin{array}{l}\text { Percent time spent in } \\
\text { cavity } \beta_{f z 2}(\mathrm{SE})\end{array}$ & $\begin{array}{c}\text { Percent time spent } \\
\text { begging by a larva } \\
\beta_{f z 3}(\mathrm{SE})\end{array}$ & $\begin{array}{l}\text { Number of larvae } \\
\text { begging } \beta_{f z 4}(\mathrm{SE})\end{array}$ \\
\hline Dispersal weight & $\begin{array}{c}-.002(.005) \\
P=.729\end{array}$ & $\begin{array}{l}.005(.004) \\
P=.3654\end{array}$ & $\begin{array}{c}.018(.003) \\
P<.001\end{array}$ & $\begin{array}{c}-.009(.004) \\
P=.021\end{array}$ \\
\hline $\begin{array}{l}\text { Duration of larval stage } \\
\text { on the resource }\end{array}$ & $\begin{array}{c}-.394(.171) \\
P=.025\end{array}$ & $\begin{array}{c}.275(.156) \\
P=.083\end{array}$ & $\begin{array}{c}-.008(.113) \\
P=.941\end{array}$ & $\begin{array}{c}.280(.139) \\
P=.049\end{array}$ \\
\hline $\begin{array}{l}\text { Duration of larval stage } \\
\text { off the resource }\end{array}$ & $\begin{array}{c}-.572(.308) \\
P=.069\end{array}$ & $\begin{array}{c}.782(.282) \\
P=.007\end{array}$ & $\begin{array}{c}-.163(.205) \\
P=.430\end{array}$ & $\begin{array}{c}.192(.250) \\
P=.447\end{array}$ \\
\hline $\begin{array}{l}\text { Duration of pupal } \\
\text { stage }\end{array}$ & $\begin{array}{c}.449(.162) \\
P=.008\end{array}$ & $\begin{array}{c}-.169(.150) \\
P=.264\end{array}$ & $\begin{array}{c}.127(.108) \\
P=.244\end{array}$ & $\begin{array}{c}-.279(.132) \\
P=.039\end{array}$ \\
\hline
\end{tabular}

Note: Independent variables (parent and offspring behaviors) were standardized to a mean of 0 , SD $=1$, to allow direct comparisons of strength of selection. Bold type $=$ significant with Bonferroni correction.

to the larvae (Scott and Gladstein 1993). Personal foraging increases the potential future fitness of parents even though it decreases offspring fitness (Scott and Gladstein 1993; A. J. Moore, S. Musa, and P. T. Smiseth, unpublished data). Thus, $N$. vespilloides parents are competitors for the food resource as well as caretakers to their offspring, resulting in potential parent-offspring conflict.

\section{Integration of Selection and Inheritance: The Adaptive Nature of Parent and Offspring Behavior Expressed in Interactions}

If offspring benefit by both begging more and receiving more provisioning, why don't all offspring and parents show high levels of begging and provisioning? Why should the genetic correlation be positive? Agrawal et al. (2001) suggest that the negative correlation in the burrower bugs they study is a coadaptation because there will be combinations of high begging/low provisioning or low begging/high provisioning that result in equal offspring fitness on average. A negative correlation equalizes the benefits and costs of both. Under this reasoning, a positive correlation would seem to be not adaptive given that high/ high combinations would be superior to low/low combinations.

Agrawal et al.'s (2001) argument is based on the results of a model of stabilizing selection on the interacting phenotypes (Wolf and Brodie 1998). Selection is often more complex than pure stabilizing selection when there are parent-offspring interactions (Kölliker 2003). Our results support a pattern of complex selection in N. vespilloides that is often directional and sometimes includes nonlinear components. Further, the sign of the selection or perfor- mance gradient depended on which traits were examined in our study. We suggest that the complex pattern of selection that we found arising from parent-offspring interactions in $N$. vespilloides, combined with genetic covariances among traits with contrasting effects, provides insights into why parent-offspring covariances may be difficult to predict. It also allows us to suggest that the parent and offspring traits in N. vespilloides are coadapted regardless of the sign of the genetic correlations.

Our study suggests that in N. vespilloides, parents can have negative effects on their offspring by spending time in the cavity without feeding them. However, parents cannot feed their offspring unless they enter the cavity. Likewise, a parent feeds only begging larvae, but there can be competition for food that has negative effects on offspring performance. The pattern of genetic covariances suggests that parents that are likely to spend more time feeding themselves while in the cavity also spend more time feeding their offspring and vice versa. Families that are likely to have high levels of larval begging also have higher numbers of begging larvae and increased competition. This suggests that there are multiple stable combinations of behavior in N. vespilloides.

The reasons for the positive genetic correlations between all traits are not known, but positive correlation can arise if there are trade-offs elsewhere in the system (Houle 1991; Arnold 1992). Our results suggest that in N. vespilloides, one such trade-off may arise because a short larval period is most strongly influenced by parental provisioning while growth (mass) is most strongly influenced by offspring begging. The presence of family members, however, may constrain the evolution toward offspring optima because the different parental and offspring behaviors are not in- 
dependent of each other. Balancing the positive and negative effects of interactions with relatives therefore requires a trade-off because larger individuals require a longer development time because individuals cannot easily grow quickly and grow to be large. A positive coadaptation overcomes some of the costs associated with larval competition and the presence of parents because the parents will provision the offspring more often; that is, positive directional selection will result in increased provisioning even if more larvae beg and create more competition. If, in contrast, the parents do not associate with the offspring, the offspring will not beg, in which case both the costs and the benefits of begging and the presence of parents will be minimized.

Parent-offspring interactions are complex interacting phenotypes and are both targets and agents of selection (Moore et al. 1998). Most models (Wolf and Brodie 1998; Wolf 2000) have focused on levels of offspring begging and parental provisioning, but we suggest that this may be too simplistic a view. Our results suggest that all behaviors that are potentially functionally related should be taken into account when studying social selection and evolution in parent-offspring interactions.

\section{Acknowledgments}

We thank M. Gibbs and S. Musa for assistance with husbandry. C. Klingenberg, T. Moore, J. Wolf, three anonymous reviewers, and especially B. Brodie and M. Kölliker provided helpful discussions and comments on earlier versions of the manuscript that greatly clarified our ideas and presentation. This research was supported by a National Environment Research Council (NERC) postgraduate fellowship to J.E.L. and a NERC grant to A.J.M.

\section{Literature Cited}

Agrawal, A. F., E. D. Brodie III, and J. Brown. 2001. Parentoffspring coadaptation and the dual genetic control of maternal care. Science 292:1710-1712.

Anduaga, S., and C. Huerta. 2001. Effect of parental care on the duration of larval development and offspring survival in Nicrophorus mexicanus Matthews (Coleoptera: Silphidae). Coleopterists Bulletin 55:264-271.

Arnold, S. J. 1983. Morphology, performance and fitness. American Zoologist 23:347-361.

—. 1992. Constraints on phenotypic evolution. American Naturalist 140(suppl.):S85-S107.

Beeler, A. E., C. M. Rauter, and A. J. Moore. 1999. Pheromonally mediated mate attraction by males of the burying beetle Nicrophorus orbicollis: alternative calling tactics conditional on both intrinsic and extrinsic factors. Behavioral Ecology 10:578-584.

Brodie, E. D., III, A. J. Moore, and F. J. Janzen. 1995.
Visualizing and quantifying natural selection. Trends in Ecology \& Evolution 10:313-318.

Cheverud, J. M., and A. J. Moore. 1994. Quantitative genetics and the role of the environment provided by relatives in behavioral evolution. Pages 67-100 in C. R. B. Boake, ed. Quantitative genetic studies of behavioral evolution. University of Chicago Press, Chicago.

Clutton-Brock, T. H. 1991. The evolution of parental care. Princeton University Press, Princeton, N.J.

Crook, J. H. 1972. Sexual selection, dimorphism and social organization in the primates. Pages 231-281 in B. Campbell, ed. Sexual selection and the descent of man, 1871-1971. Aldine, Chicago.

Eggert, A. K., and J. K. Müller. 1997. Biparental care and social evolution in burying beetles: lessons from the larder. Pages 216-236 in J. C. Choe and B. J. Crespi, eds. The evolution of social behavior in insects and arachnids. Cambridge University Press, Cambridge.

Eggert, A. K., M. Reinking, and J. K. Müller. 1998. Parental care improves offspring survival and growth in burying beetles. Animal Behaviour 55:97-107.

Hager, R., and R. A. Johnstone. 2003. The genetic basis of family conflict resolution in mice. Nature 421:533535.

Houle, D. 1991. Genetic covariance of fitness correlates: what genetic correlations are made of and why it matters. Evolution 45:630-648.

Kocárek, P. 2001. Diurnal activity rhythms and nice differentiation in a carrion beetle assemblage (Coleoptera: Silphidae) in Opava, the Czech Republic. Biological Rhythm Research 32:431-438.

Kölliker, M. 2003. Estimating mechanisms and equilibria for offspring begging and parental provisioning. Proceedings of the Royal Society of London B 270(suppl.): 110-113.

Kölliker, M., and H. Richner. 2001. Parent-offspring conflict and the genetics of offspring solicitation and parental response. Animal Behaviour 62:395-407.

Kölliker, M., M. W. G. Brinkhof, P. Heeb, P. S. Fitze, and H. Richner. 2000. The quantitative genetic basis of offspring solicitation and parental response in a passerine bird with biparental care. Proceedings of the Royal Society of London B 267:2127-2132.

Lande, R., and S. J. Arnold. 1983. The measurement of selection on correlated characters. Evolution 37:12101226.

Martin, P., and P. Bateson. 1986. Measuring behaviour: an introductory guide. Cambridge University Press, Cambridge.

Moore, A. J., E. D. Brodie III, and J. B. Wolf 1997. Interacting phenotypes and the evolutionary process. I. direct and indirect genetic effects of social interactions. Evolution 51:1352-1362. 
Moore, A. J., J. B. Wolf, and E. D. Brodie III. 1998. The influence of direct and indirect genetic effects on the evolution of behavior: social and sexual selection meet maternal effects. Pages 22-41 in T. A. Mousseau and C. W. Fox, eds. Maternal effects as adaptations. Oxford University Press, Oxford.

Moore, A. J., K. F. Haynes, R. F. Preziosi, and P. J. Moore. 2002. The evolution of interacting phenotypes: genetics and evolution of social dominance. American Naturalist 160(suppl.):S186-S197.

Müller, J. K., and A.-K. Eggert. 1990. Time-dependent shifts between infanticide and parental behavior in female burying beetles: a mechanism of indirect motheroffspring recognition. Behavioral Ecology and Sociobiology 27:11-16.

Rauter, C. M., and A. J. Moore. 1999. Do honest signalling models of offspring solicitation apply to insects? Proceedings of the Royal Society of London B 266:16911696.

- 2002a. Evolutionary importance of parental care performance, food resources, and direct and indirect genetic effects in a burying beetle. Journal of Evolutionary Biology 15:407-417.

- 2002b. Quantitative genetics of growth and development time in the burying beetle Nicrophorus pustulatus in the presence and absence of post-hatching parental care. Evolution 56:96-110.

- In press. Time constraints and trade-offs among parental care behaviours: effects of brood size, sex, and loss of mate. Animal Behaviour.

Rosenblatt, J. S., and C. T. Snowdon, eds. 1999. Parental care: evolution, mechanisms, and adaptive significance. Academic Press, San Diego, Calif.

Scott, M. P. 1998. The ecology and behavior of burying beetles. Annual Review of Entomology 43:595-618.

Scott, M. P., and D. Gladstein. 1993. Calculating males? the duration of parental care in burying beetles. Evolutionary Ecology 7:362-378.

Smiseth, P. T., and A. J. Moore. 2002. Does resource avail- ability affect offspring begging and parental provisioning in a partially begging species? Animal Behaviour 63: 577-585.

- 2004a. Complex family dynamics: joint effects of social interactions and resource availability. Behavioral Ecology (in press).

. 2004b. Signalling of hunger when offspring forage by both begging and self-feeding. Animal Behaviour (in press).

Smiseth, P. T., C. T. Darvell, and A. J. Moore. 2003. Partial begging: an empirical model for the early evolution of offspring signaling. Proceedings of the Royal Society of London B 270:1773-1777.

Sokal, R., and F. J. Rohlf. 1995. Biometry. 3d ed. W. H. Freeman, New York.

Stockley, P., and G. A. Parker. 2002. Life history consequences of mammal sibling rivalry. Proceedings of the National Academy of Sciences of the USA 99:1293212937.

Wade, M. J. 1998. The evolutionary genetics of maternal effects. Pages 5-21 in T. A. Mousseau and C. W. Fox, eds. Maternal effects as adaptations. Oxford University Press, Oxford.

West-Eberhard, M. J. 1983. Sexual selection, social competition, and speciation. Quarterly Review of Biology 58:155-183.

Wolf, J. B. 2000. Gene interactions from maternal effects. Evolution 54:1882-1898.

Wolf, J. B., and E. D. Brodie III. 1998. The coadaptation of parental and offspring characters. Evolution 52:299308.

Wolf, J. B., E. D. Brodie III, and A. J. Moore. 1999. Interacting phenotypes and the evolutionary process. II. selection resulting from social interactions. American Naturalist 153:254-266.

Wright, J., and M. L. Leonard, eds. 2002. The evolution of begging: competition, cooperation and communication. Kluwer Academic, Dordrecht.

Associate Editor: Ben C. Sheldon 